\title{
Signatures of knee osteoarthritis in women in the temporal and fractal dynamics of human gait
}

Citation for published version (APA):

Vangeneugden, J., Verlaan, L., Oomen, P., Liu, W-Y., Peters, M., Natour, N., Emans, P., \& Meijer, K. (2020). Signatures of knee osteoarthritis in women in the temporal and fractal dynamics of human gait. Clinical Biomechanics, 76, [105016]. https://doi.org/10.1016/j.clinbiomech.2020.105016

Document status and date:

Published: 01/06/2020

DOI:

10.1016/j.clinbiomech.2020.105016

Document Version:

Publisher's PDF, also known as Version of record

Document license:

Taverne

Please check the document version of this publication:

- A submitted manuscript is the version of the article upon submission and before peer-review. There can be important differences between the submitted version and the official published version of record.

People interested in the research are advised to contact the author for the final version of the publication, or visit the DOI to the publisher's website.

- The final author version and the galley proof are versions of the publication after peer review.

- The final published version features the final layout of the paper including the volume, issue and page numbers.

Link to publication

\footnotetext{
General rights rights.

- You may freely distribute the URL identifying the publication in the public portal. please follow below link for the End User Agreement:

www.umlib.nl/taverne-license

Take down policy

If you believe that this document breaches copyright please contact us at:

repository@maastrichtuniversity.nl

providing details and we will investigate your claim.
}

Copyright and moral rights for the publications made accessible in the public portal are retained by the authors and/or other copyright owners and it is a condition of accessing publications that users recognise and abide by the legal requirements associated with these

- Users may download and print one copy of any publication from the public portal for the purpose of private study or research.

- You may not further distribute the material or use it for any profit-making activity or commercial gain

If the publication is distributed under the terms of Article $25 \mathrm{fa}$ of the Dutch Copyright Act, indicated by the "Taverne" license above, 
Lecture

\title{
Signatures of knee osteoarthritis in women in the temporal and fractal dynamics of human gait
}

\author{
Joris Vangeneugden $^{\mathrm{a}, \mathrm{b}, 1}$, Loek Verlaan ${ }^{\mathrm{a}, 1, *}$, Pieter Oomen ${ }^{\mathrm{c}}$, Wai-Yan Liu ${ }^{\mathrm{c}}$, Marloes Peters ${ }^{\mathrm{a}}$, \\ Nicole Natour ${ }^{\mathrm{d}}$, Pieter Emans ${ }^{\mathrm{a}}$, Kenneth Meijer ${ }^{\mathrm{c}}$ \\ ${ }^{a}$ Department of Orthopedic Surgery, CAPHRI School for Public Health and Primary Care, Maastricht University Medical Center, P.O. Box 5800, 6202, AZ, Maastricht, the \\ Netherlands \\ ${ }^{\mathrm{b}}$ Department of Psychiatry \& Neuropsychology, Division of Translational Neuroscience, Maastricht University, the Netherlands \\ ${ }^{\mathrm{c}}$ Department of Human Movement Sciences, NUTRIM School for Nutrition, Toxicology and metabolism, Maastricht University Medical Center, P.O. Box 616, 6200, MD, \\ Maastricht, the Netherlands \\ ${ }^{\mathrm{d}}$ Department of Nuclear Medicine and Radiology, Maastricht University Medical Center, the Netherlands
}

A R T I C L E I N F O

\section{Keywords:}

Knee osteoarthritis

Obesity

Accelerometry

Activity pattern

\begin{abstract}
A B S T R A C T
Background: Osteoarthritis of the knee is characterized by progressive cartilage deterioration causing pain and function loss. Symptoms develop late with limited disease-modifying opportunities. Osteoarthritis is a major cause of immobility, with a higher prevalence above 60 years. This age-related increase in prevalence is further amplified by the female gender. Imaging and biochemical analyses for detection of osteoarthritis of the knee are expensive and labor-intensive. Continuous movement tracking could aid in detecting onset and/or worsening of symptoms.

Methods: We used portable technology to investigate kinematic differences in female patients with knee osteoarthritis, weight-matched healthy female volunteers and obese female patients with osteoarthritis of the knee. Knee osteoarthritis was established radiographically and corroborated using magnetic resonance imaging. Findings: The total amount, type and level of activity did not differ significantly between groups. The temporal activity pattern during the day was however significantly different with a bimodal signature in healthy volunteers only. Sequence analyses revealed more time to recuperate after dynamic activity in both patient groups. Analysis of walking bouts revealed significant differences in stride interval dynamics, indicative of gait naturalness, only in healthy volunteers. Temporal activity, sequence and walking patterns were independent of body weight.

Interpretation: We thus provide for the first-time evidence of temporal specific kinematic signatures in amount and quality of movement also in stride interval dynamics between people with and without osteoarthritis of the knee independent of body weight. These findings could allow early and non-intrusive diagnosis of osteoarthritis enabling concordant treatment.
\end{abstract}

\section{Introduction}

Osteoarthritis of the knee (KNOA; KNee OsteoArthritis) is one of the leading causes of global disability (Bijlsma et al., 2011) and the most common reason for pain in older adults with a significant individual and economic burden (Britton, 2009; Felson, 2006). It is estimated that between $20 \%$ and $30 \%$ of adults and elderly suffer from this condition (Yoshimura et al., 2017). The demographic change ahead of us, i.e. increasing average age of men and women, and lifestyle habits, i.e. increasing obesity, will only aggravate the impact of this disease
(Richmond et al., 2013). Being the largest weight-bearing joint, the knee is most affected by wear and tear and biomechanical load (FowlerBrown et al., 2015; Verhaar, 2008).

Current operative approaches for KNOA have experienced enormous improvements over the last couple of years (Bijlsma et al., 2011). However, KNOA is a disease of multifactorial origin, starting as a preclinical condition that can become very advanced before it becomes symptomatic due to the avascular and noninnervated nature of cartilage (Ryd et al., 2015), cf. the pre-osteoarthritis stage. Biochemical analyses (Heinegard and Saxne, 2011) and imaging (Eckstein et al.,

\footnotetext{
* Corresponding author.

E-mail address: 1.verlaan@mumc.nl (L. Verlaan).

${ }^{1}$ Equal contribution.
} 
2011; Huang et al., 2011; Kokkonen et al., 2012) to examine this stage are however not conclusive, expensive and invasive. Neuromuscular exercises and proprioceptive training have been successful in preventing or at least slowing down KNOA (Roos and Arden, 2016). Still on a global scale, approximately 1.5 million total knee arthroplasty (TKA) surgeries, the end-stage of KNOA, are performed (Chu et al., 2012; Leta et al., 2015; Singh, 2011).

The pathomechanics of clinically manifest KNOA is well described under restricted laboratory settings using accelerometers or motioncapturing systems (Alkjaer et al., 2015; Cho et al., 2015; Clermont and Barden, 2016) however a good understanding of subtle and small changes in behavior requires long-term and continuous monitoring of movement in a daily setting. Activity patterns are typically probed using questionnaires, however questionnaires are very susceptible to subjectivity (Skender et al., 2016).

Accelerometers have been used in a number of diverse fields (Annegarn et al., 2012; Baskerville et al., 2017; Cain et al., 2013; Lipperts et al., 2017; Noorkoiv et al., 2014; Scheer et al., 2017; Senden et al., 2011; Tabak et al., 2012; Taraldsen et al., 2012). They are less expensive, less complex to use and mimic realistic settings better than gait analysis laboratories, yet they produce more objective and detailed data than subjective interpretation of movement parameters. With regard to KNOA not much is known about kinematic signatures typical for the disease.

Most studies monitor activity with the goal to detect motion parameters capable of discriminating between healthy and pathological gaits (Bolink et al., 2016; Kirchner et al., 2014; Tabak et al., 2012). A lot more information can and should be extracted from these rich and ecologically valid data sets. Studies on the gait of healthy subjects have shown great potential of detailed motion analyses, proficiency in discerning a number of different action categories (Lugade et al., 2014) and the capability to determine the smoothness (Menz et al., 2003), rhythm (Menz et al., 2003), stability (Doi et al., 2013), harmony (Iosa et al., 2016) and naturalness of locomotion (Stergiou et al., 2006). Our goal was to document in great detail the effect of osteoarthritis of the knee on macro- and mesoscale temporal patterns and on complex gait characteristics using unconstrained, continuous and long-term monitoring.

We set out to investigate kinematic signatures and activity patterns distinctive for KNOA, measured continuously for the duration of a full week under unconstrained daily conditions. This was done by attaching triaxial accelerometers, capable of detecting positional displacement in three spatial orthogonal axes, on the non-affected femur of patients suffering from KNOA as assessed clinically and radiological compared with healthy volunteers. Only women aged between 50 and 65 years were included in study, as KNOA prevalence is highest in this group. According to the World Health Organization, OA is the fourth leading cause of immobility, with a prevalence of $18 \%$ for women and $9.6 \%$ for men aged above 60 years (Woolf and Pfleger, 2003). Prevalence of knee $\mathrm{OA}$ increases with age, especially above 50 years. This age-related increase in prevalence is further amplified by the female gender (Arden and Nevitt, 2006). Given the modulatory effect of weight we also included a group of obese KNOA patients. The rationale was that a meticulous description of potential distinctive signatures in KNOA could then be used in future studies and clinical practice to detect early-OA in healthy subjects allowing the rapid initiation of adequate preventive treatments.

The goal of our study was to examine differences in activity patterns, temporal, sequence and fractal dynamics between patients suffering from osteoarthritis of the knee and matched healthy participants following continuous, long-term (one week) and unconstrained monitoring at home, taking body weight into account.

\section{Methods}

\subsection{Subjects}

This study included three groups: healthy controls (BMI: 20-25 $\mathrm{kg} \mathrm{m}^{-2}$ ), lean KNOA (BMI: $20-25 \mathrm{~kg} \mathrm{~m}^{-2}$ ) and obese KNOA (BMI: $30-40 \mathrm{~kg} \mathrm{~m}^{-2}$ ). Only women aged between 50 and 65 years were included, as KNOA prevalence is highest in this group. The upper aged limit was adopted to prevent inclusion of participants at high risk of having comorbidities (e.g. type II diabetes mellitus, osteoporosis, dementia, cardiovascular disease). OA patients having a Kellgren Lawrence (KL) score between 1 and 3 at the medial tibiofemoral site were included.

All subjects participated in a larger study, i.e. the KNOA study referring to "Knee Osteoarthritis", coordinated at the Maastricht University Medical Center (MUMC+). The KNOA study consisted of monitoring physical activity over a longer time span, i.e. one week, while subjects were instructed to engage in regular daily activities. Radiological assessment of KNOA was done by means of the Kellgren and Lawrence classification system (Kellgren and Lawrence, 1957) and further corroborated by magnetic resonance imaging using the MRI Osteoarthritis Knee Score, i.e. MOAKS (Hunter et al., 2011).

Exclusion criteria were any inflammatory arthritis, trauma, OA at any other joint in the lower extremities including patellofemoral OA and tibiofemoral OA on the lateral site, anterior cruciate ligament injury, medial and collateral ligament injury, and psychiatric illness according to the Diagnostic and Statistical Manual of Mental Disorders classification criteria for psychiatric illnesses (patients were excluded when diagnoses were present in their medical files). Healthy women were non-obese, did not meet the exclusion criteria, and did not have knee OA according to the American College of Rheumatology classification criteria (Altman et al., 1986).

The study was approved by the Medical Ethical Committee Maastricht University Medical Centre and all subjects gave their informed consent. All procedures were in accordance with the ethical standards of the institutional and national research committee and with the 1964 Helsinki declaration and its later amendments.

\subsection{Imaging analyses: radiography and magnetic resonance imaging}

Radiographic imaging was used to evaluate knee cartilage and knee OA status. Presence of knee OA was assessed from X-ray images by the Kellgren-Lawrence (KL) knee score (Kellgren and Lawrence, 1957). Subjects having a score between 1 and 3 at the medial tibiofemoral site were included in this study. The X-ray images were evaluated doubleblind by two independent orthopedic surgeons.

To more accurately assess cartilage health in all study groups, Magnetic Resonance Imaging (MRI) was performed using a 3 T Philips Intera Scanner (Philips Medical Systems, Best, The Netherlands). Cartilage health was evaluated based on the MRI Osteoarthritis Knee Score (MOAKS) (Hunter et al., 2011). For a more detailed description of imaging procedures, we refer to Verlaan et al. (Verlaan et al., 2018)

\subsection{Equipment}

Accelerations were measured using a three-dimensional accelerometer $(49 \times 40 \times 14 \mathrm{~mm}$; length, width $\mathrm{x}$ height; weight: $30 \mathrm{~g})$, i.e. KXSD9 tri-axis Digital Accelerometer (http://www.microelectronicos.com/datasheets/KXSD9-2050.pdf, n.d.) with a Texas Instruments microcontroller capable of monitoring positional displacements expressed in amount of gravitational inertial force $(\mathrm{g})$ in anteroposterior, mediolateral and vertical or cranial-caudal directions (Senden et al., 2011). The accelerometer was secured midway between patella and spina iliaca anterior superior using adhesive hypoallergic tape to reduce extraneous movements as much as possible. Data were sampled at $25 \mathrm{~Hz}$ and stored on a local internal memory of $2 \mathrm{~GB}$. The 
unit was powered by a Lithium Ion battery capable of recording approximately two weeks at abovementioned sampling frequency (Senden et al., 2011).

\subsection{Protocol}

Accelerometers were positioned and instructions were given at the policlinic of orthopedic surgery. Subjects were instructed to wear the accelerometer at all times during a full week to ten days when a new appointment was made to remove the apparatus. Accelerometers were waterproof and adhesive tape strong enough for the full duration. Importantly, subjects were not primed or influenced in any way to move different than usual. Instructions for correct reattaching the accelerometer when necessary were also provided.

\subsection{Data analysis}

Raw data was downloaded to a PC using commercially available software packages (IDEEQ from Maastricht Instruments Inc.). Files were converted to readable .bin files and imported into Matlab (Mathworks, Natick, MA, USA) for further processing and analyses. Analyses and algorithms applied were largely in line with previously published work from our laboratory (Annegarn et al., 2012; Senden et al., 2011). Kinematic traces were converted to conventional coordinates following International Society of Biomechanics (ISB) gait guidelines (Wu et al., 2005). Parameters of interest were: different levels of activity, i.e. sitting/lying, standing and dynamically active and differentiations of dynamically activity from bottom to top respectively low, medium and vigorous activity. Average physical activity per subject and per group. Fluctuations of average physical activity during the course of the day. Differences in time to recuperate after activity and complex fluctuations in gait pattern using detrended fluctuation analyses. A detailed description on all procedures and algorithms written in Matlab can be found as supplementary material to this paper (Supplementary Methods). All data is made available to the general public and can be obtained by contacting the corresponding author.

\subsection{Statistical analysis}

Non-parametric statistics, i.e. Kruskal-Wallis $\mathrm{H}$ tests, equivalent to one-way ANOVAs, with Mann-Whitney $U$ tests, to test for pairwise significance were run on data with only one factor and limited data entries, i.e. when running hypotheses of one variable between groups. $\mathrm{N}$-way ANOVAs were run on hypotheses containing more than one factor, e.g. the temporal signature of average activity over the course of the day between groups. Significant F-statistics were followed up with Bonferonni-corrected pairwise post-hoc tests. All analyses were performed in Matlab and custom-scripts were made if not available in the library or online repositories. A $P$-value of $\leq 0.05$ was considered significant. Bootstrap analysis was performed on the temporal activity patterns between groups by creating an empirical distribution constructed by drawing 10,000 random samples $(n=10)$ with replacement. Bootstrap confidence intervals were set at $95 \%$.

\section{Results}

\subsection{Subjects characteristics}

Thirty-two subjects were included in this study, divided over three study groups. The first group consisted of healthy volunteers $(n=11)$, the second group of body weight matched subjects suffering from KNOA with Kellgren-Lawrence (KL) scores ranging from 1 to $3(n=11$; mean KL 2) and the third group of obese subjects with KNOA and Kellgren-Lawrence scores 1 to 3 ( $\mathrm{n}=10$; mean $\mathrm{KL} 2.43$; comparison between KL-scores of the two KNOA groups, Mann-Whitney $U$ test, $U=1.78, P=0.1826$ ). Furthermore, there was no significant
Table 1

Anthropometric measurements and imaging characteristics. An asterisk indicates a significant difference of the variable between the three groups (Kruskal-Wallis test). Significant differences between groups, tested with concordant Mann-Whitney $U$ tests, are between groups 1 and 2 with group 3 on weight and BMI, between group 1 and groups 2 and 3 on MOAKS and KL. There was no significant association between KL-grades in the two different OA groups (group 2 and group 3) (Chi-square statistic $=1.29, P=0.52$ ).

\begin{tabular}{|c|c|c|c|c|}
\hline \multirow[t]{4}{*}{ Parameter } & Group 1 & Group 2 & Group 3 & \multirow[t]{4}{*}{$P$} \\
\hline & $\begin{array}{l}\text { Healthy } \\
\text { volunteers }\end{array}$ & $\begin{array}{l}\text { Lean KNOA } \\
\text { patients }\end{array}$ & $\begin{array}{l}\text { Obese KNOA } \\
\text { patients }\end{array}$ & \\
\hline & $\mathrm{n}=11$ & $\mathrm{n}=11$ & $\mathrm{n}=10$ & \\
\hline & Mean (SD) & Mean (SD) & Mean (SD) & \\
\hline Age (years) & $57.6(4.5)$ & $60.2(4.7)$ & 59.9 (4) & 0.3 \\
\hline Height (m) & $1.67(0.05)$ & $1.66(0.06)$ & $1.63(0.1)$ & 0.34 \\
\hline Weight (kg) & $64.1(5.74)$ & $66.9(6.3)$ & $84.9(13.44)$ & $<0.001 *$ \\
\hline BMI $\left(\mathrm{kg} / \mathrm{m}^{2}\right)$ & $22.83(1.12)$ & $24.36(2.16)$ & $31.27(2.07)$ & $<0.001^{*}$ \\
\hline KL (grade) & n.a. & $2(0.63)$ & $2.3(0.67)$ & 0.18 \\
\hline$(1 ; 2 ; 3)$ & & $(2 ; 7 ; 2)$ & $(1 ; 5 ; 4)$ & \\
\hline MOAKS (grade) & $0.29(0.36)$ & $1.01(0.66)$ & $1.04(0.75)$ & 0.95 \\
\hline
\end{tabular}

association between KL-grades in the two different OA groups (group 2 and group 3) (Chi-square statistic $=1.29, P=0.52$ ). The MOAKS between the healthy volunteers and the two KNOA groups, lean and obese, were also significantly different (mean MOAKS 0.29 ( $n=8$ ) vs. $1.01(\mathrm{n}=9)$ and $1.04(\mathrm{n}=9)$ respectively; Mann-Whitney $U$ tests, both $P$ 's $=0.02$ ), but not between the two patient groups (Mann-Whitney $U$ test, $P=0.95$ ). Obesity thus has no influence on the severity of the osteoarthritis as evidenced by magnetic resonance imaging. Please note that we did not obtain MRI data from all subjects, i.e. group $1=8 / 11$, group $2=9 / 11$ and group $3=9 / 10$. Average BMIs were 22.83, 24.36 and $31.27 \mathrm{~kg} / \mathrm{m}^{2}$ respectively (Kruskal-Wallis test, $H=20.02$, $P<0.001$; post-hoc Mann-Whitney $U$ test showed significant differences between group 3 and other 2 groups only). Groups were also matched according to age (57.6, 60.2 and 59.9 years; Kruskal-Wallis test, $H=2.43, P=0.02966)$. Full anthropometric measurements can be found in Table 1 .

\subsection{Continuous monitoring and classification of kinematics}

The accelerometers we employed lasted 7-10 days without recharging and sampling quality was good and stable during the entire duration as evidenced by a randomly chosen epoch of $20 \mathrm{~min}$ from a random subject (Fig. 1a). Fig. 1b represents a 2-min zoomed-in section from mid-epoch. Action classification results are indicated by the full black line above the kinematic traces. During active episodes we further looked at the intensity of activity, plotted as dots above the classification line.

For each subject we visualized the average activity level per hour in color plots (Fig. 1c) to discern potential temporal patterns of activity that could discriminate between the three groups. Red colors represent hours with lots of activity, blue colors the opposite. As such, day-night cycli are easily discriminable (Tracy et al., 2014).

Next, we applied correlation analyses using the 24-h activity patterns to look for potential patterns between all recorded days of all subjects (Fig. 1d) and between an averaged 24-h curve per subject within or between groups (Fig. 1e). The average correlation index per day within each group was $0.39,0.38$ and 0.33 respectively (unbalanced one-way ANOVA, $F=32.94, P<0.001$ ) with a significantly lower index for the obese KNOA patients compared to the other two groups $(P<0.001$, Bonferonni-corrected). This points to the fact that within the obese KNOA group larger differences between subjects exist. 
a

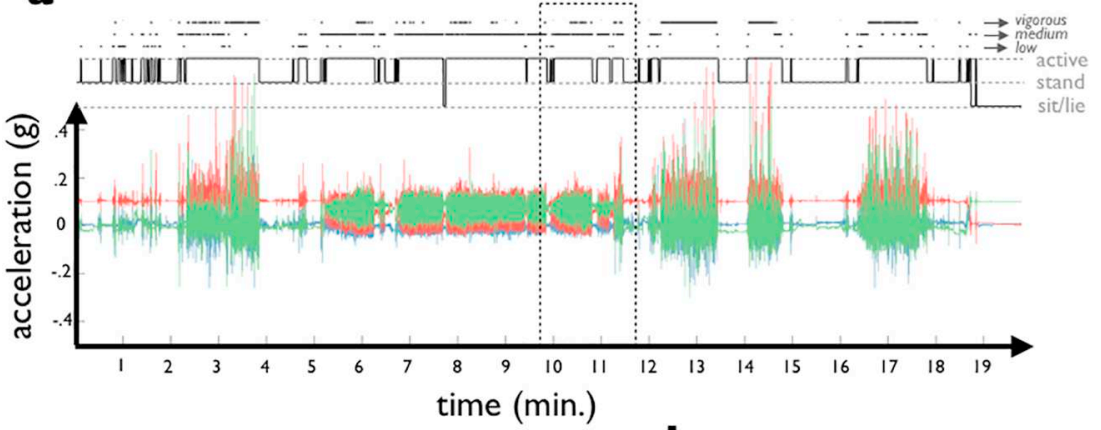

C

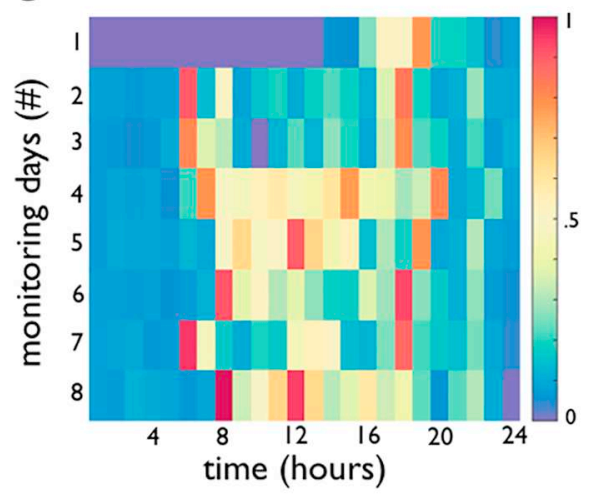

d

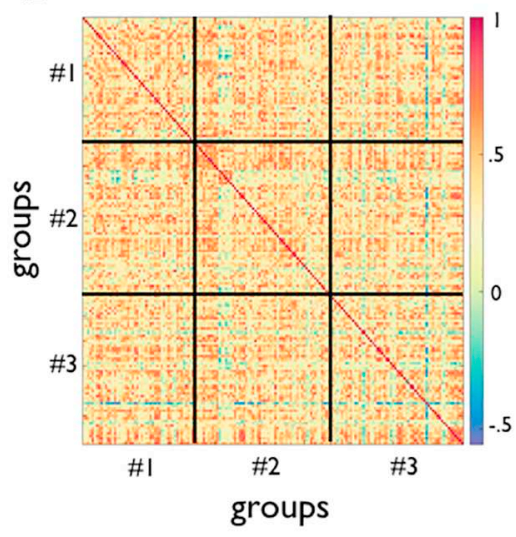

b

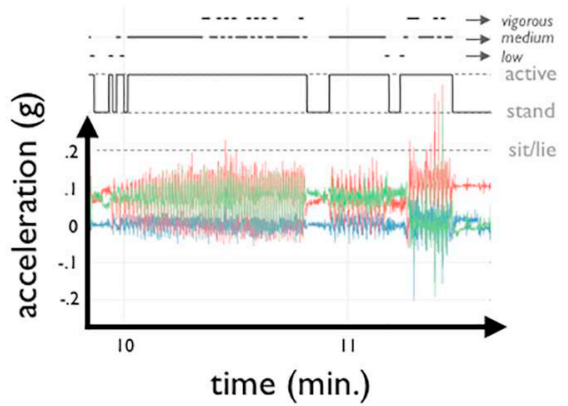

e

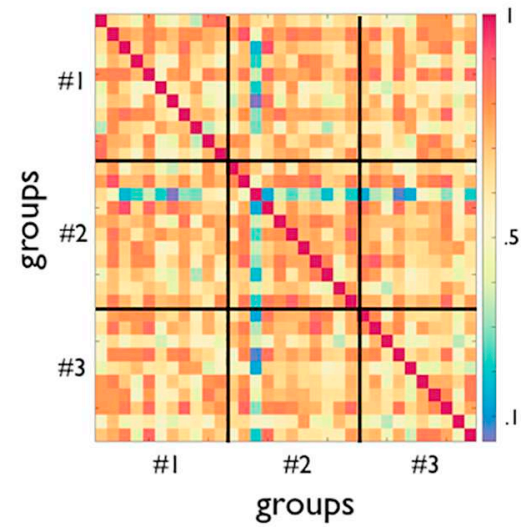

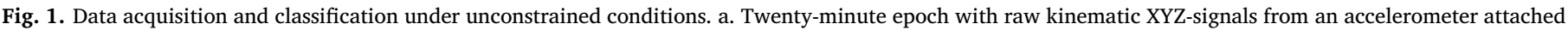

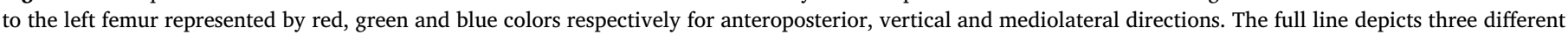

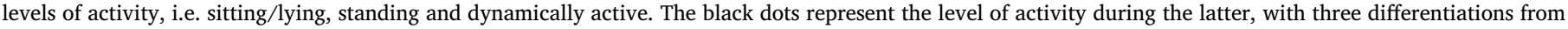

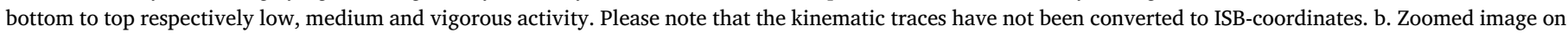

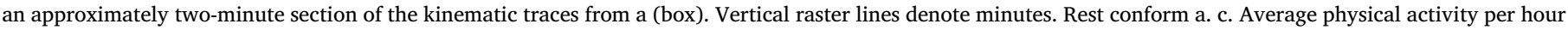

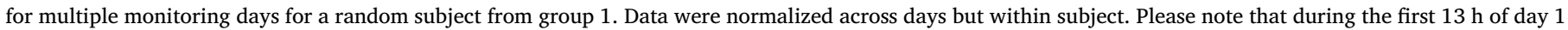

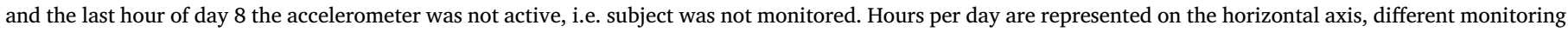

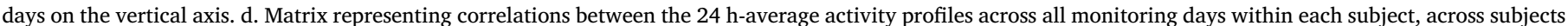

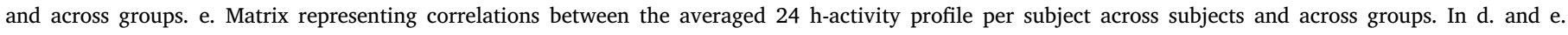

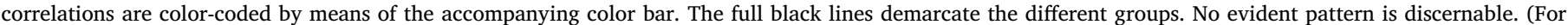
interpretation of the references to color in this figure legend, the reader is referred to the web version of this article.)

\subsection{OA does not affect total amount, type nor level of activity}

Next, we looked at potential differences in temporal signatures of activity patterns between groups. For each subject in each group we obtained one average temporal signature, represented in Fig. 2a. Two subjects from the first group and one subject from the third group were excluded due to insufficient full monitoring days ( $<5$ days). We either normalized the temporal signatures to the maximum activity pattern of each subject individually (Fig. 2a upper panels) or to the maximum activity within the whole group (Fig. 2a lower panels). The former gives an idea on the absolute fluctuations in activity during the day per subject, while the latter gives a more realistic and relative indication of fluctuation patterns over groups. In total there are no differences between groups in activity pattern over days (Fig. 2b; Kruskal-Wallis test, $H=2.1, P>0.05)$. Subjects in all three groups moved on average equally over days, although healthy subjects tended to move a bit more than lean subjects with KNOA (SMA group $1=0.2754$ units vs. SMA group $2=0.2443$ units) who in turn tended to move a bit more than obese subjects with KNOA (SMA group $3=0.2277$ units), however all effects were not significant. Similar results were obtained when testing the per-subject normalized data.

The average amount of time (proportions) spent sitting or lying, standing or being dynamically active during waking hours (Fig. 2c left panel) did not reveal significant differences between groups (respective scores group 1: 0.6, 0.34 and 0.06; group 2: $0.55,0.38$ and 0.07; group
3: 0.6, 0.35 and 0.05; unbalanced two-way ANOVA, $F=0.21$, $P=0.99$ ). The level of dynamic activity, expressed as frequencies, was also not significantly different (respective scores group 1: 2.02, 19.15 and 0.87; group 2: 2.04, 18.54 and 1.2; group 3: 3.15, 17 and 0.79; unbalanced two-way ANOVA, $F=0.39, P=0.68$; see Fig. $2 \mathrm{c}$ right panel; notice that proportions do not add up to 1 given that during some hours subjects did not express any physical activity, e.g. sitting at a desk for longer than $1 \mathrm{~h}$ ).

Based on these analyses it is clear that KNOA, in our sample, does not affect the total amount of physical activity, type of activity, nor the level of activity.

\subsection{Lack of bimodal activity patterns during the course of the day in $O A$}

However, eyeballing the color plots in Fig. 2a, qualitative differences between groups seem to be present. To get a better idea on temporal fluctuations, we plotted and directly compared the temporal signatures of the three groups (Fig. 3). Temporal patterns over days differed significantly (two-way unbalanced ANOVA, $F=18.56$, $P<0.001$ ) with lower levels of activity in the afternoon from 3 to 5 P.M. for the KNOA patients, independent of body weight. Also, temporal activity patterns in the morning, at 12 A.M. for the obese patients and from 11 A.M. to 1 P.M. for the lean KNOA group differed with the healthy subjects. All post-hoc pairwise tests were performed using Bonferonni correction. Notwithstanding the lack of a general significant 
a

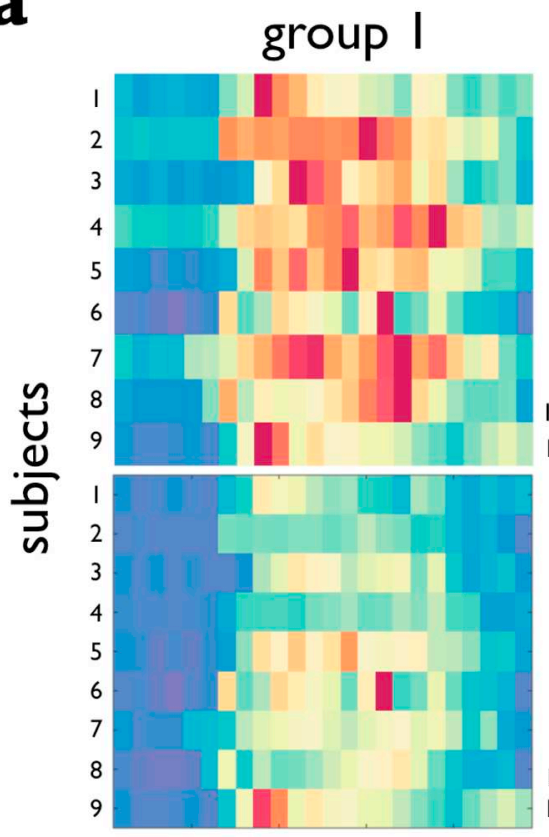

group 2

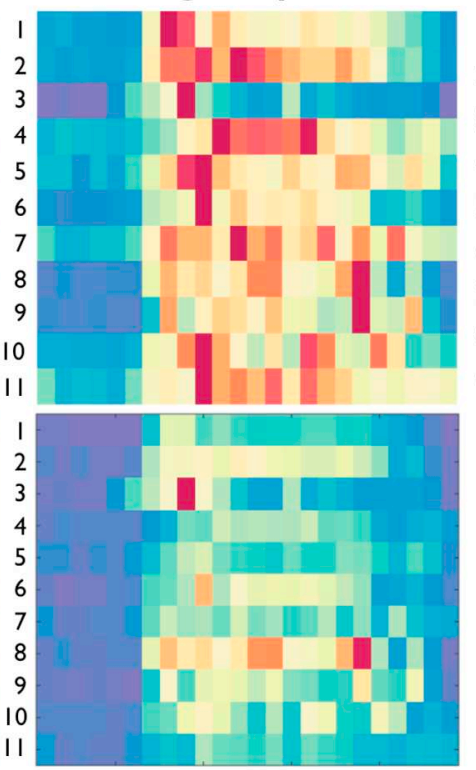

group 3

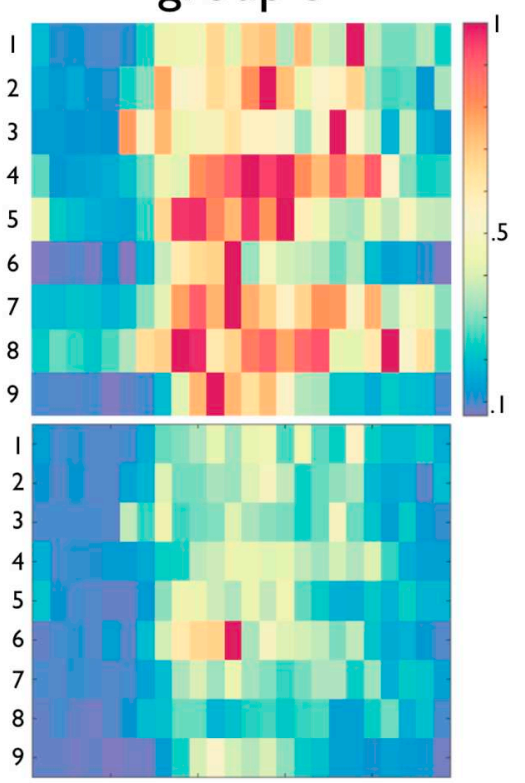

b

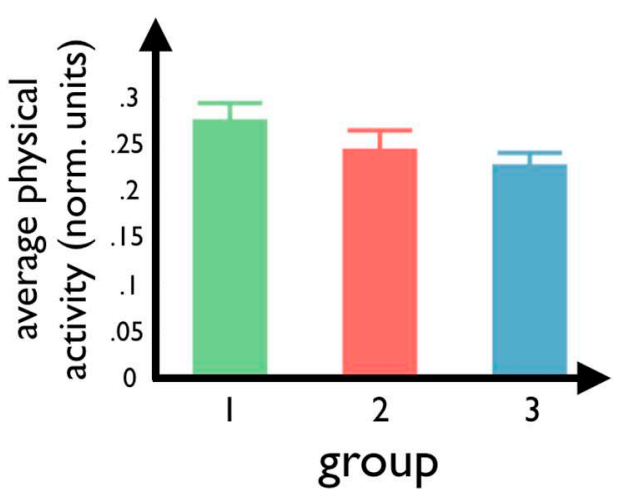

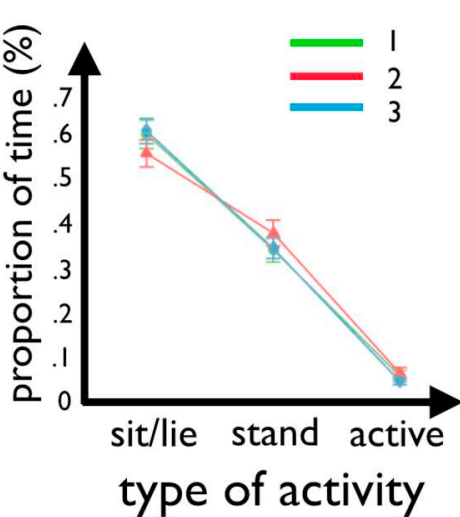

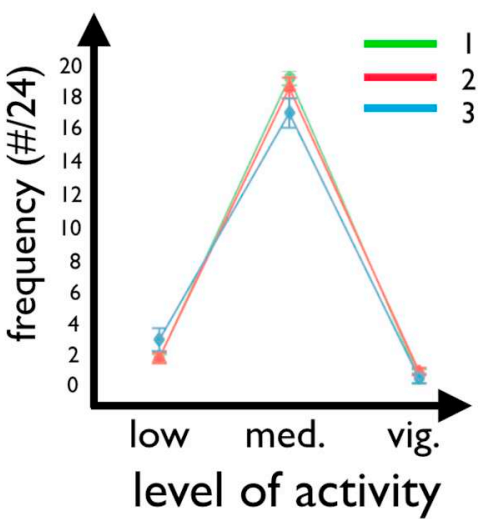

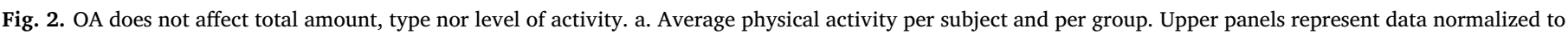

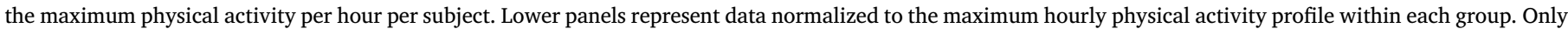

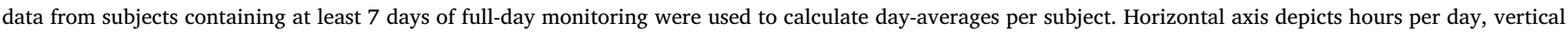

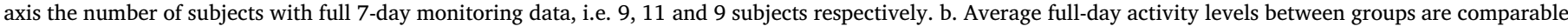

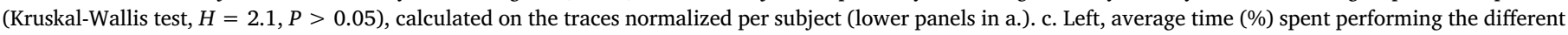

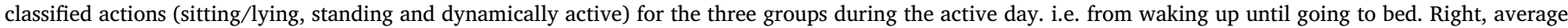

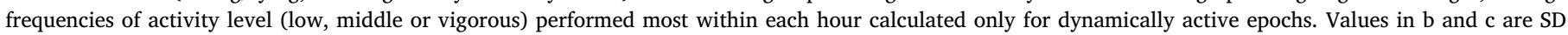
standard error of the mean.

effect in physical activity over days (Fig. 2b), examining temporal signatures in more detail reveals group-specific patterns with a more bimodal type of activity-level in the healthy group. Results were corroborated by bootstrap analysis with significantly lower levels of activity for the KNOA patients in the afternoon, from 3 to 5 P.M., and vice versa for the healthy subjects (all $P s<0.05$ ). Furthermore, the increased activity levels at 12 A.M. was significant for the healthy subjects and significantly lowered in the lean (both $P$ s $<0.05$ ), but not the obese patients. However, the differences at 11 A.M. and 1 P.M. were not significant between groups. Thus, the main finding of increased temporal activity patterns in healthy subjects as compared to subjects suffering from osteoarthritis in the early afternoon, i.e. from 3 to 5 P.M., was not due to small sample sizes.

\subsection{Increased time to recuperate in OA following locomotion but not standing}

We were interested in potential differences in time to recuperate after such events. Instead of looking at large spans of time, i.e. 1-h epochs, we divided the temporal kinematic traces in bouts of $5 \mathrm{~min}$ and subjected it to our action classification algorithm. Following a standing epoch, no significant differences were observed within the 30-min interval between groups (repeated measures two-factor ANOVA, $F=1$, $P=0.37$ ) (Fig. 4a). Following an active epoch, significant differences between groups were observed (repeated measures two-factor ANOVA, $F=5.18, P<0.01$ ), albeit without any significant pairwise comparison (Fig. 4b). There thus seems to exist a general trend for more rest, i.e. sitting or standing, after an active period in patients with KNOA as compared to healthy subjects. 


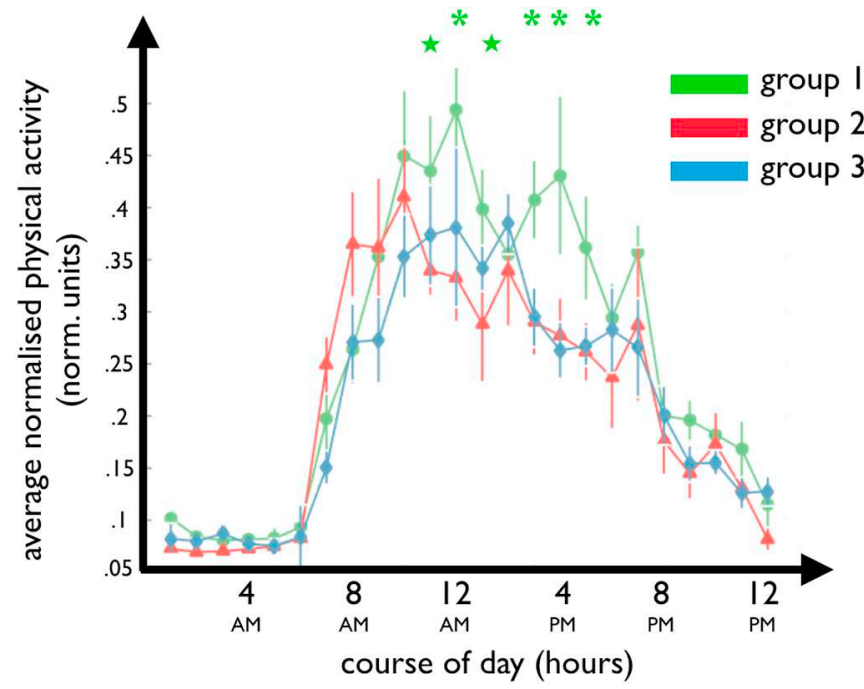

Fig. 3. Different temporal activity patterns between groups. Fluctuations of average physical activity during the course of the day are different for the three groups. Calculated on 9, 11 and 9 subjects respectively. A bimodal signature is present in activity patterns of healthy subjects (group 1) with significant peak activities in late morning and late afternoon. Values are SD standard error of the mean. Green asterisks and stars represent significant pairwise comparison at $P^{\prime}$ s $<0.01$ between group 1 and group $2+3$ and group 1 and group 2 respectively. (For interpretation of the references to color in this figure legend, the reader is referred to the web version of this article.)

\subsection{Reduced stride-to-stride fluctuations in $O A$}

An important yet subtle observation in the analysis of gait dynamics has provided insight into pathological alterations in the physiology of gait (Hausdorff, 2007; Hausdorff et al., 1997). Close examination of gait revealed complex fluctuations in gait pattern and more specifically in stride-to-stride fluctuations previously considered to be mere noise. Short- and long-term autocorrelations of these non-stationary signals can be unraveled using detrended fluctuation analyses (DFA), a fractal analysis technique, in line with extensive research in cardiology (Goldberger et al., 2002).

Following the extraction of walking bouts (Annegarn et al., 2012), a previously validated algorithm based on the filtered anteroposterior acceleration signal using integration processes and peak detection algorithms (Zijlstra and Hof, 2003) was applied to detect left and right steps. Based on these left-right steps we were able to look at a number of walking parameters influenced by osteoarthritis or obesity between the three groups.

Mean step time (medians $607 \mathrm{~ms}, 637 \mathrm{~ms}$ and $697 \mathrm{~ms}$ respectively; Kruskal-Wallis test, $H=1.32, P=0.5165$; Fig. 5a), average of mean stride time left and right (medians $1.0145 \mathrm{~s}, 1.0752 \mathrm{~s}$ and $1.1932 \mathrm{~s}$ respectively; Kruskal-Wallis test, $H=1.29, P=0.5165$; Fig. 5b) and harmonic ratios, indicative of the smoothness and rhythm of gait (anteroposterior direction: medians 2.1869, 1.8506 and 2.2793 respectively; Kruskal-Wallis test, $H=0.19, P=0.9114$; vertical direction: medians 2.5486, 2.1427 and 2.5075 respectively; Kruskal-Wallis test, $H=0.18, P=0.9129$; lateromedial direction: medians 0.5627 , 0.5194 and 0.4218 respectively; Kruskal-Wallis test, $H=1.46$, $P=0.4808$; Fig. 5c; all harmonic ratios expressed in amplitudes), were similar across the three groups.

However, the DFA index, a parameter of locomotor function looking at stride-to-stride time fluctuations as an indicator for naturalness or good health of gait, was significantly different between groups (medians of slope $\alpha 0.8138,0.6695$ and 0.6424 respectively; Kruskal-Wallis test, $H=7.58, P<0.05$ ), with significant differences between the group of healthy subjects and both patients groups with KNOA, but independent of BMI (Mann-Whitney $U$ test, $U s$ 2.07, 2.85 and 0.04 between group $1 \& 2$, group $1 \& 3$ and group $2 \& 3 ; P<0.05, P<0.05$ and $P=0.9719$ respectively).

\section{Discussion}

To the best of our knowledge this study is the first in its kind to document in great detail the effect of osteoarthritis of the knee on macro- and mesoscale temporal patterns and on complex gait characteristics using unconstrained, continuous and long-term monitoring.

Within the domain of KNOA only a handful of studies have been carried out previously using accelerometers, yet this study differed notably from these studies in a number of ways. A first study by Liu and colleagues (Liu et al., 2016) looked into the effect of KNOA on physical activity and symptoms change over the course of a year. This study however lacked a control group of matched healthy volunteers. BMI as potential confounder for OA (Zheng and Chen, 2015) was not considered in their design and motion analyses only allowed discriminating light from moderate-to-vigorous activity.

A second study by Staab and colleagues (Staab et al., 2014) investigated gait parameters more in detail using spectral analyses and a combination of accelerometry and motion capturing by an optoelectronic system. This study did contain a control group of osteoarthritisfree subjects although not matched according to age or weight. According to our results the latter non-matched variable should not resort too much effect, yet the former variable can have serious implications on detailed gait parameters (Tracy et al., 2014). Moreover, we only found a significant difference in the presence of more long-range strideto-stride time interval correlations (DFA) in healthy subjects compared to patients. Staab and colleagues (Staab et al., 2014) did find differences in gait velocity, cadence and symmetry, possibly pointing to the fact that their groups were more diverse than our groups. The fact that we only found a specific and well-defined difference between patients

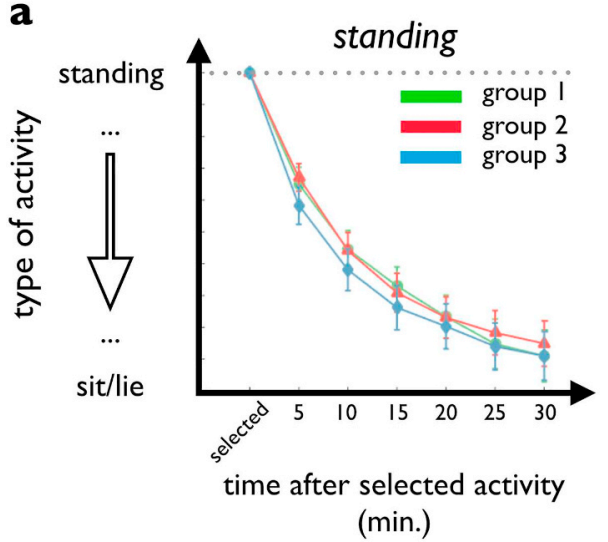

Fig. 4. Increased time to recuperate in OA following locomotion but not standing. Sequential consequences after standing (a) or being dynamically active (b) for at least $5 \mathrm{~min}$ on the activity type in the subsequent $30 \mathrm{~min}$, divided in $5 \mathrm{~min}$ sections. Values range from 1 to 3 , i.e. $1=$ sitting/lying, 2 = standing and $3=$ dynamic activity. Two-way ANOVAs not significant in $a$, but factor 'group' was significant in $b(P<0.01)$. 
a

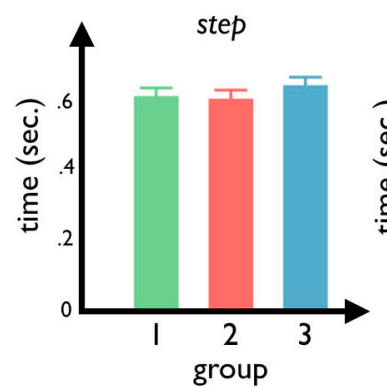

b

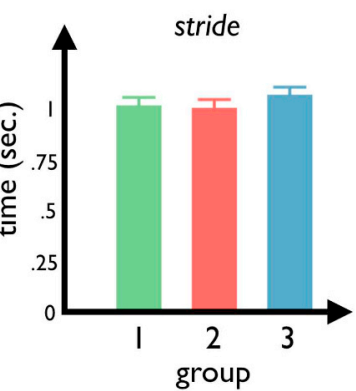

C

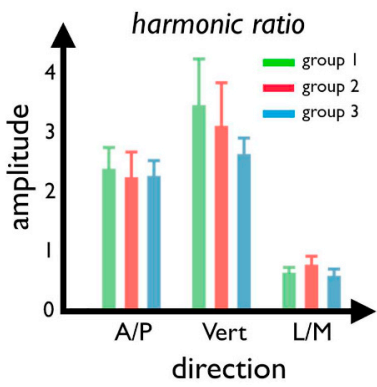

d

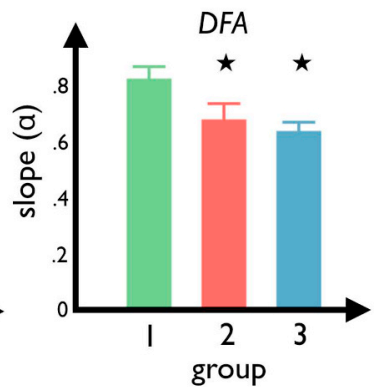

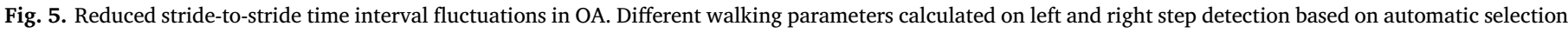

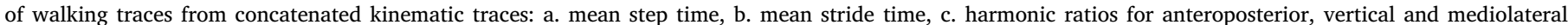
accelerations and $d$. detrended fluctuation analysis (DFA). Black stars denote significant $p$-values $\left(P^{\prime}\right.$ s $\left.<0.05\right)$.

with and without KNOA in the domain of naturalness of gait and not on any other gait parameter could provide evidence for a more defined demarcation of KNOA in our study.

Recently, both Alkjaer and colleagues (Alkjaer et al., 2015) and Clermont and Barden (Clermont and Barden, 2016) did not find significant differences in DFA between patients with and without KNOA. The DFA for subjects from our healthy group was in range of previously reported DFA values from their and other studies (our DFA: 0.81 vs. DFAs from other studies: $0.72,0.77,0.88$ and 0.76 , the former two DFAs from the abovementioned studies, the latter two from (Gates et al., 2007) and (Kobsar et al., 2014) respectively). The DFA values of our KNOA patients were lower than reported by Alkjaer et al. (Alkjaer et al., 2015) and Clermont and Barden (Clermont and Barden, 2016), i.e. 0.67 and 0.64 vs. 0.80 and 0.77 respectively. This could be due to a number of reasons. Firstly, we used longer kinematic traces for our stride-to-stride calculations (on average $40 \mathrm{~min}$. vs. $10 \mathrm{~min}$. in both studies). Secondly and more importantly we monitored subjects under unconstrained and natural conditions, thus being less susceptible to experiment-desired outcomes, e.g. forced locomotion during the monitoring phase in a laboratory setting (Dingwell and Cusumano, 2010). The variety in severity of KNOA as measured by the Kellgren and Lawrence scale was similar and is unlikely to account for the observed differences (Kiss, 2011). We do not think that the nature of the concatenated signal used in our analyses could account for these discrepancies (Kirchner et al., 2014).

Another important addition to these studies is the fluctuating activity pattern during the course of the day in healthy volunteers but not KNOA patients. This finding is completely new within the field of KNOA but has been observed before in COPD patients (Tabak et al., 2012) and could also provide opportunities for early detection and initiation of neuromuscular and proprioceptive training programs (Roos and Arden, 2016). However, we were not able to relate this difference in temporal activity pattern to the severity of the underlying cartilage damage (KLscores) due to the low number of subjects in our study. Nor were we able to relate this finding to the level of experienced pain since we did not collect these data.

Furthermore, our sequence analyses pointing to more time needed for recuperation in KNOA patients after being active, but not standing still, is also completely new and could also be used as a tool in early detection. We must however emphasize that pairwise post-hoc tests did reveal trends, but not significance, between healthy volunteers and KNOA patients. A more extensive study with more subjects in all groups could potentially yield significant results when analyzing kinematic data to detect sequence effects.

We are mindful of the fact that we only had a limited amount of subjects in our three groups. Moreover, we only included females. It is known that KNOA increases with age, especially in people above 50 years and that this increment is amplified in women (Arden and Nevitt, 2006). Although our subjects were within this age range, we do not think our data could not be extrapolated to the other gender or other age groups. A more extensive study with more subjects (including males), longer and more monitoring periods could strengthen our results.

More importantly, we realize that our study did not incorporate experienced pain as predictor or moderator to manifest behavior. Fukutani and colleagues (Fukutani et al., 2016) have recently shown that patients with late KNOA (KL-scale 3 and 4) ascend and descend stairs less and avoid sit-to-stand transitions more than patients with early KNOA (KL-scale 1 and 2), a finding associated with experienced pain. To account for these observations, we recently recorded detailed motion data via an optoelectronic motion capturing system, of the same subjects included in this study performing the actions investigated by Fukutani and colleagues (Fukutani et al., 2016), i.e. walking on a flat surface (Verlaan et al., submitted), ascending and descending stairs (Verlaan et al., 2019) and sit-to-stand (Verlaan et al., 2018) and vice versa.

\section{Conclusion}

This study systematically examined differences in activity patterns, temporal, sequence and fractal dynamics between patients suffering from osteoarthritis of the knee and matched healthy participants following continuous, long-term (one week minimum) and unconstrained monitoring at home, taking body weight into account.

In sum, the results highlight the importance of continuous monitoring of movement patterns in daily settings in order to discriminate at the behavioral level patients with and without KNOA. As such, these subtle yet significant differences in temporal dynamics, i.e. temporary decrease in activity in early afternoon and more time needed to recuperate after physical activity, and fractal dynamics, i.e. with reduced naturalness of gait, between patients with knee osteoarthritis and healthy controls could be used as behavioral markers for early detection and/or worsening of symptoms in KNOA (Chu et al., 2012; Ryd et al., 2015). These behavioral markers are measured easily, rapidly and noninvasively and can operate in concert with biomarkers and (non)-invasive imaging techniques to signal potential underlying structural damage in subjects prone to development of OA, i.e. presence of risk factors (Ryd et al., 2015).

\section{Funding}

This research did not receive any specific grant from funding agencies in the public, commercial, or not-for-profit sectors.

\section{Declaration of Competing Interest}

None of the authors have competing interests to declare. 


\section{Acknowledgments}

We would like to express our gratitude to the expert assistance of Dr. W. Bijnens in data analyses, Dr. V. Groen and Dr. A. Witlox for helpful discussions and finally Prof. L. van Rhijn for creating a positive and collaborative environment between departments of Orthopeadic Surgery, Movement Sciences and Radiology. J. Vangeneugden was a Medical Student at the University of Maastricht (physician - clinical researcher) and a Marie Curie IEF post-doctoral fellow at the time of the study. Finally, we are very grateful to all the subjects who participated in the KNOA protocol.

\section{Appendix A. Supplementary data}

Supplementary data to this article can be found online at https:// doi.org/10.1016/j.clinbiomech.2020.105016.

\section{References}

Alkjaer, T., et al., 2015. Gait variability and motor control in people with knee osteoarthritis. Gait Posture 42, 479-484.

Altman, R., Asch, E., Bloch, D., Bole, G., Borenstein, D., Brandt, K., et al., 1986. Development of criteria for the classification and reporting of osteoarthritis. Classification of osteoarthritis of the knee. Diagnostic and Therapeutic Criteria Committee of the American Rheumatism Association. Arthritis Rheum. 29, 1039-1049.

Annegarn, J., et al., 2012. Differences in walking pattern during 6-min walk test between patients with COPD and healthy subjects. PLoS One 7, e37329.

Arden, N., Nevitt, M.C., 2006. Osteoarthritis: epidemiology. Best Pract. Res. Clin. Rheumatol. 20, 3-25.

Baskerville, R., Ricci-Cabello, I., Roberts, N., Farmer, A., 2017. Impact of accelerometer and pedometer use on physical activity and glycaemic control in people with type 2 diabetes: a systematic review and meta-analysis. Diabet. Med. 34, 612-620.

Bijlsma, J.W., Berenbaum, F., Lafeber, F.P., 2011. Osteoarthritis: an update with relevance for clinical practice. Lancet. 377, 2115-2126.

Bolink, S.A.A.N., et al., 2016. Validity of an inertial measurement unit to assess pelvic orientation angles during gait, sit-stand transfers and step-up transfers: comparison with an optoelectronic motion capture system. Med. Eng. Phys. 38, 225-231.

Britton, R., 2009. The economic burden of osteoarthritis. Am. J. Manag. Care 15, 230-235.

Cain, K.L., Sallis, J.F., Conway, T.L., Van Dyck, D., Calhoon, L., 2013. Using accelerometers in youth physical activity studies: a review of methods. J. Phys. Act. Health $10,437-450$.

Cho, Y., Kim, M., Lee, W., 2015. Effect of proprioceptive training on foot posture, lower limb alignment, and knee adduction moment in patients with degenerative knee osteoarthritis: a randomized controlled trial. J. Phys. Ther. Sci. 27, 371-374.

Chu, C.R., Williams, A.A., Coyle, C.H., Bowers, M.E., 2012. Early diagnosis to enable early treatment of pre-osteoarthritis. Arthritis Res. Ther. 14, 212-222.

Clermont, C.A., Barden, J.M., 2016. Accelerometer-based determination of gait variability in older adults with knee osteoarthritis. Gait Posture 50, 126-130.

Dingwell, J.B., Cusumano, J.P., 2010. Re-interpreting detrended fluctuation analyses of stride-to-stride variability in human walking. Gait Posture 32, 348-353.

Doi, T., et al., 2013. The harmonic ratio of trunk acceleration predicts falling among older people: results of a 1-year prospective study. J. Neuroeng. Rehabil. 10, 1-6.

Eckstein, F., et al., 2011. Clinical, radiographic, molecular and MRI-based predictors of cartilage loss in knee osteoarthritis. Ann. Rheum. Dis. 70, 1223-1230.

Felson, D.T., 2006. Clinical practice. Osteoarthritis of the knee. N. Engl. J. Med. 354, $841-848$.

Fowler-Brown, A., et al., 2015. The mediating effect of leptin on the relationship between body weight and knee osteoarthritis in older adults. Arthritis Rheum. 67, 169-175.

Fukutani, N., et al., 2016. Knee pain during activities of daily living and its relationship with physical activity in patients with early and severe knee osteoarthritis. Clin. Rheumatol. 35, 2307-2316.

Gates, D.H., Su, J.L., Dingwell, J.B., 2007. Possible biomechanical origins of the longrange correlations in stride intervals of walking. Physica A. 380, 259-270.

Goldberger, A.L., et al., 2002. Fractal dynamics in physiology: alterations with disease and aging. Proc. Natl. Acad. Sci. U. S. A. 99, 2466-2472.

Hausdorff, J.M., 2007. Gait dynamics, fractals and falls: finding meaning in the stride-tostride fluctuations of human walking. Hum. Mov. Sci. 26, 555-589.

Hausdorff, J.M., Edelberg, H.K., Mitchell, S.L., Goldberger, A.L., Wei, J.Y., 1997. Increased gait unsteadiness in community-dwelling elderly fallers. Arch. Phys. Med. Rehabil. 78, 278-283.

Heinegard, D., Saxne, T., 2011. The role of the cartilage matrix in osteoarthritis. Nat. Rev. Rheumatol. 7, 50-56.

http://www.microelectronicos.com/datasheets/KXSD9-2050.pdf.

Huang, Y.P., Wang, S.Z., Saarakkala, S., Zheng, Y.P., 2011. Quantification of stiffness change in degenerated articular cartilage using optical coherence tomography-based air-ject indentation. Connect. Tissue Res. 52, 433-443.

Hunter, D.J., et al., 2011. Evolution of semi-quantative whole joint assessment of knee OA: MOAKS (MRI Osteoarthritis Knee Score). Osteoarthr. Cartil. 19, 990-1002.

Iosa, M., et al., 2016. Stability and harmony of gait in patients with subacute stroke. J. Med. Biol. Eng. 36, 635-643.

Kellgren, J.H., Lawrence, J.S., 1957. Radiological assessment of rheumatoid arthritis. Ann. Rheum. Dis. 16, 485-493.

Kirchner, M., Schubert, P., Liebherr, M., Haas, C.T., 2014. Detrended fluctuation analysis and adaptive fractal analysis of stride time data in Parkinson's disease: stitching together short gait trials. PLoS One 1-6 e85787.

Kiss, R.M., 2011. Effect of severity of knee osteoarthritis on the variability of gait parameters. J. Electromyogr. Kinesiol. 21, 695-703.

Kobsar, D., Olson, C., Paranjape, R., Barden, J.M., 2014. The validity of gait variability and fractal dynamics obtained from a single, body-fixed triaxial accelerometer. J. Appl. Biomech. 30, 343-347.

Kokkonen, H.T., et al., 2012. Delayed computed tomography arthrography of human knee cartilage in vivo. Cartilage. 3, 334-341.

Leta, T.H., Lygre, S.H., Skredderstuen, A., Hallan, G., Furnes, O., 2015. Failure of aseptic revision total knee arthroplasties. Acta Orthop. 86, 48-57.

Lipperts, M., van Laarhoven, S., Senden, R., Heyligers, I., Grimm, B., 2017. Clinical validation of a body-fixed 3D accelerometer and algorithm for activity monitoring in orthopaedic patients. J. Orthop. Trans. 11, 19-29.

Liu, S.H., et al., 2016. Objectively measured physical activity and symptoms change in knee osteoarthritis. Am. J. Med. 129, 497-505.

Lugade, V., Fortune, E., Morrow, M., Kaufman, K., 2014. Validity of using tri-axial accelerometers to measure human movement - part I: posture and movement detection. Med. Eng. Phys. 36, 169-176.

Menz, H.B., Lord, S.R., Fitzpatrick, R.C., 2003. Acceleration patterns of the head and pelvis when walking on level and irregular surfaces. Gait Posture 18, 35-46.

Noorkoiv, M., Rodgers, H., Price, C.I., 2014. Accelerometer measurement of upper extremity movement after stroke: a systematic review of clinical studies. J. Neuroeng. Rehabil. 11, 144-155.

Richmond, S.A., et al., 2013. Are joint injury, sport activity, physical activity, obesity, or occupational activities predictors for osteoarthritis? A systematic review. J. Orthop. Sports Phys. Ther. 43, 515-519.

Roos, E.W., Arden, N.K., 2016. Strategies for the prevention of knee osteoarthritis. Nat. Rev. Rheumatol. 12, 92-101.

Ryd, L., et al., 2015. Pre-osteoarthritis: definition and diagnosis of an elusive clinical entity. Cartilage. 6, 156-165.

Scheer, J.K., et al., 2017. Initial experience with real-time continuous physical activity monitoring in patients undergoing spine surgery. Clin. Spine Surg. 30, 1434-1443.

Senden, R., Grimm, B., Meijer, K., Savelberg, H., Heyligers, I.C., 2011. The importance to including objective functional outcomes in the clinical follow up of total knee arthroplasty patients. Knee. 18, 306-311.

Singh, J.A., 2011. Epidemiology of knee and hip arthroplasty: a systematic review. Open Orthop. 5, 80-85.

Skender, S., et al., 2016. Accelerometry and physical activity questionnaires - a systematic review. BMC Public Health 16, 515-525.

Staab, W., et al., 2014. Accelerometer and gyroscope based gait analysis using spectral analysis of patients with osteoarthritis of the knee. J. Phys. Ther. Sci. 26, 997-1002.

Stergiou, N., Harbourne, R., Cavanaugh, J., 2006. Optimal movement variability: a new theoretical perspective for neurologic physical therapy. J. Neurol. Phys. Ther. 30, $120-129$.

Tabak, M., et al., 2012. Telemonitoring of daily activity and symptom behavior in patients with COPD. Int. J. Telemed. Appl. 438736, 1-8.

Taraldsen, K., Chastin, S.F., Riphagen, I.I., Vereijken, B., Helbostad, J.L., 2012. Physical activity monitoring by use of accelerometer-based body-worn sensors in older adults: a systematic literature review of current knowledge and applications. Maturitas 71, 13-19.

Tracy, D.J., et al., 2014. Separating bedtime rest from activity using waist or wrist-worn accelerometers in youth. PLoS One 9, 1-9.

Verhaar, J.A.N., 2008. Aandoeningen van de knie. In: Verhaar, van Mourik (Eds.), Leerboek Orthopedie. Bohn Stafleu van Loghum, pp. 381-407.

Verlaan, L., Boekesteijn, R.J., Oomen, P.W., Liu, W.-Y., Peters, M.J.M., Witlox, M.A., et al., 2018. Biomechanical alterations during sit-to-stand transfer are caused by a synergy between knee osteoarthritis and obesity. Biomed. Res. Int. 2018, 7.

Verlaan, L., Boekesteijn, R., Oomen, P.W., Liu, W.Y., Peters, M.J.M., Emans, P.J., van Rhijn, L.W., Meijer, K., 2019. Knee adduction moments are not increased in obese knee osteoarthritis patients during stair negotiation. Gait Posture 73, 154-160.

Woolf, A.D., Pfleger, B., 2003. Burden of major musculoskeletal conditions. Bull. World Health Organ. 81, 646-656.

Wu, G., et al., 2005. ISB recommendation on definitions of joint coordinate systems of various joints for the reporting of human joint motion - part II: shoulder, elbow, wrist and hand. J. Biomech. 38, 981-992.

Yoshimura, N., Muraki, S., Nakamura, K., Tanaka, S., 2017. Epidemiology of the locomotive syndrome: the research on osteoarthritis/osteoporosis against disability study 2005-2015. Mod. Rheumatol. 27, 1-7.

Zheng, H., Chen, C., 2015. Body mass index and risk of knee osteoarthritis: systematic review and meta-analysis of prospective studies. BMJ Open 5, 1-8.

Zijlstra, W., Hof, A.L., 2003. Assessment of spatio-temporal gait parameters from trunk accelerations during human walking. Gait Posture 18, 1-10. 